\title{
A Smart Mobile Pregnancy Management and Awareness System for Saudi Arabia
}

\author{
https://doi.org/10.3991/ijim.v12i5.9005 \\ Mohammed Alotaibi( $\left.{ }^{\bowtie}\right)$, Maram Albalawi, Layan Alwakeel \\ University of Tabuk, Tabuk, Saudi Arabia \\ mmalotaibi@ut.edu.sa
}

\begin{abstract}
Over the years, physicians and other medical personnel have lamented the ever-increasing prevalence of physical inactivity and low awareness amongst pregnant women all over the world and especially in Saudi Arabia. The physicians argue that pregnant women are continuously placing their lives at risk by ignoring physical activity and low awareness. Similarly, the pregnant women who rarely participate in physical activity and awareness programs are accredited with risking the lives of their babies. As a result, professional medics have been trying to come up with innovative strategies that can promote physical activity and improve health awareness amongst the pregnant women for a long time. In recent years, the world has experienced a massive shift towards technology-based solutions for nearly every aspect of life.
\end{abstract}

Keywords - mHealth; pregnancy; healthcare informatics; physical activity; wearable sensors

In the health industry, mobile Health has become a key trend as tech companies liaise with medical practitioners in pursuit of technological solutions to diverse health concerns. With this understanding, the current paper provides a vivid analysis of a newly-developed technological system designed to facilitating communication between pregnant women and healthcare providers, increase interactions amongst pregnant women, as well as motivate them to increase their participation in physical activities and remote monitoring of their babies. Unlike most mHealth initiatives that provide specific functionalities, the current system is an all-inclusive system offering a combination of all functionalities available through individual pregnancy monitoring systems. The newly-developed system aims to empower pregnant with enough awareness of pregnancy and also to do more physical activity using advanced technology of internet of things and mobile health technology specially in remote areas.

\section{Introduction}

Pregnancy denotes the period between conception and delivery. In normal conditions, it takes roughly 40 weeks. It is difficult to ascertain the statistics and rates of pregnancy because of the inclusion of miscarriages as one of the key outcomes of 
pregnancy. According to data gathered and published by the Central Intelligence Agency World Factbook, the birth rate in Saudi Arabia pregnancy rate stands at 18.3 births / 1,000 population (1). During pregnancy, women need special care and special lifestyle to guarantee healthy life for them and their babies. Thereby, pregnant mothers require Physical Activity (PA), regular pregnancy check-up, as well as knowledge about pregnancy management to get healthier babies.

Physical Activity (PA) empowers communities to avoid diseases. The importance of PA increases during pregnancy to maintain solid health for both the women and her foetus (2). Physical activity helps pregnant women to control their weight (3)(4) as well as minimize the probability of contracting gestational diabetes and early delivery (5). Additionally, physical activity helps decrease foetal heart rate (6). However, it is shocking to note that pregnant women have the lowest rates of participation in physical activity compared to non-pregnant women (15.8 vs. $26.1 \%)(7)$. Research indicates that pregnant women usually raise their activity in the first six months. However, a large percentage of them never attain the levels of physical activity recommended by The American College of Obstetricians and Gynaecologists (ACOG), which is at least 150 minutes of physical activity every week (8). Moreover, studies show that a majority of pregnant women are totally inactive during the last three months (8). Pregnant women often argue that they could become more active if they knew the importance of PA in terms of safety and effectiveness (8).

Pregnant women also claim that they would double their activeness in physical activity if they receive guidance on how to conduct PA during prenatal care (8). Several studies also identify low educational level and poor income as obstacles that prevent pregnant women from engaging in continuous PA (8).

Over the last decade, revolution in Mobile technology has altered interaction patterns amongst people. The recent innovations in the field of mobile technology have contributed significantly to the health care industry. With the invention and implementation of mobile health technology in various aspects of health care, the world is progressing towards a future characterized by secure healthcare. Mobile health is a division of electronic health practice, which is defined as "a medical practice supported with the use of mobile technology which includes mobile phones, wireless devices, patent monitoring devices and many others" (9).

The affordability of mobile devices has significantly contributed towards better disease decision-making, strict adherence to treatments, fewer personal visits to the doctor, as well as good service experiences. Mobile health empowers individuals and institutions to conduct voluntary programs, immunization campaigns, and counselling sessions for people in remote areas. The use of text messaging is a low-cost approach for health campaigns and promotion. Mobile health technology plays an important role in improving the maternity health of women in low income areas. In particular, mobile health provide a medium for communicating with the women frequently. The idea is to keep them aware about the best practices to be observe. These include diet, schedule of check-up, vaccination for children and many others.

Mobile health has increased the percentage of safe childbirths and reduced the ratio of misconceptions. More so, mobile health has empowered specialists to measure the compliance of medics to standards of the treatment when treating patients. A major 
strength of Mobile Health is that it is less error prone and hence can be used to provide efficient healthcare solutions (9).

However, several barriers restrict the growth of mobile health technology by limiting applicability to a wider group of people. Social, economic and financial barriers have stalled the expansion of mobile health on a global perspective. Since mobile health is in its early stage of adoption, it lacks sufficient evidence that can prove that it is the most convenient health practice (10). Lack of infrastructure, widespread network coverage and availability of mobile devices are other obstacles that halt the development of mobile health. Security, confidentiality and privacy in mobile health are major concerns because absence of proper data security measures can lead to leakage of confidential and personal information (11).

\section{$2 \quad$ Related Work}

In recent years, numerous scholars have focused on the use of mobile health management and awareness systems of chronic disease (12). Studies are investigating the use of technological systems like cell phones, wireless devices and self-monitoring devices and their influence on pregnancy monitoring and awareness.

Some studies (8)(13) have targeted the effectiveness of using mobile health technology in improving and enhancing the control of pregnancy. A prime example of these studies was published in 2016 by Choi and his peers (13). The study aimed at testing the feasibility and potential efficacy of using mobile health physical activity intervention for a period of 12 weeks. 30 women, who were between 10 and 20 weeks of gestation, were selected as participants for the study. The researchers used random sampling to place the women into two distinctive groups. One group used a mobile phone app plus Fitbit while the other unit comprised of a control group using Fitbit only. Both conditions targeted steady increases in physical activity.

The trial version of the Fitbit measured PA in terms of distance, steps, estimated calories expended, and flights of stairs climbed. In the trial app, a daily message was available between $10 \mathrm{am}$ and $7 \mathrm{pm}$ while the activity diary was available after $7 \mathrm{pm}$ to midnight daily. The study concluded that it is acceptable to use mobile technologies to motivate pregnant women to increase physical activity.

Another study that considered the use of technology to encourage pregnant women to indulge in physical activity was published in (8). The study aimed at determining the efficiency of physical activity SMS messages to improve PA in pregnant women compared to the standard T4baby. The study sought to identify the most effective dose and time of the SMS to improve PA. Text4baby (T4b) is a nation-wide mobile health information service that sends text messages (SMS) to pregnant women. The study participants included 85 pregnant women aged at least 18 years old. All women had pregnancy for a duration between 8 to 16 weeks. It used FitibTM Flex to measure physical activity. The women were asked to wear it all the time. The study used mixed model analysis to analyze the collected data. The study prepared standard T4b SMS and PA SMS content and divided pregnant women into four groups. The groups differed in frequency and time of receiving SMS messages: 
1. The Standard group received three T4B SMS and two PA SMS three times a week at noon

2. Plus One group received two PA and one T4b three times a week

3. Plus Six group received one T4b and six PA one every noon

4. Plus Six group received similar service as the third group, but at the time they chose

The results of the study were as follows: 5 women dropped out of the study for different reasons, one of them was due to skin reactions to FitibTM. The remaining 80 pregnant women reported no improvement in physical activity from SMS intervention for all groups (different dose and frequency). The fourth group experienced a huge decrease in activity and a noteworthy increase in sedentary time. These results were not surprising because only three of nine studies reported improvement in physical activity in the past. However, none of these past studies were mHealth or SMS-based intervention. The study concludes there were no increases in PA regardless of frequency or time. Those that received six PA SMS/week had greater decreases in activity and greater increases in sedentary time. SMS may not be "potent" enough strategy to improve PA.

Text4baby is another study based on similar principles (2). The primary purpose of the study was to determine the optimal dose of physical activity text message (SMS) to improve the physical activity of pregnant women. The study was based on the usercentered design process and included three phases. In the first phase, literature review and interview with 15 pregnant women was conducted to understand their needs. The objective was to develop prototype text messages. In the second phase, they gained feedback about the proposed prototype using additional interviews with seven physicians comprising of five nurses and a group of 5 pregnant women. The researchers developed 168 additional text messages for this stage. The third phase involved surveying 326 pregnant women to determine the preferred dose of text messages to inform the design of the researcher's intervention.

From the technical perspective, there are so many applications in both App Store and Play Store that specialized in pregnancy control. Two independent reviewers searched in Google Play and iTunes using two key words: pregnancy and pregnant in Arabic and in English language for the applications launched during the last two years. The number of existing apps in play store was 24 and the number of existing apps in iTunes was 36 . There are nearly 17 Arabic pregnancy applications in iTunes and nearly 20 in Play Store. Independent reviewers reviewed each app description and summarized the main functions of each app and listed them in a table. At the end, each duplicated function was removed from the table to get the main common features as shown in table 1 .

We observe that most mobile applications available in Google Play and iTunes cover one or more functions that appear in table1. None of these applications include all functions together, yet these functions are crucial for proper pregnancy monitoring and awareness. To overcome this gap, we developed our mobile application to include all mentioned functions in table 1 using advanced technology like the wrist bond 
which will be explained in next sections. The current paper illustrates different goals that the application is trying to achieve. These include:

- Facilitating communication between pregnant women and healthcare providers.

- Facilitating communication amongst the pregnant women to exchange their experience.

- Increasing pregnant women's awareness using modern technology.

- Monitoring pregnant women's walking using a wrist bond sensor connected to the application as shown in System Overview section below

- Monitoring the movement of unborn baby using the kick counter belt sensor.

- Saudi Arabia is a large country and there is lack of Obstetrics and Gynecology Clinics in remote areas, so the developed system is trying to deliver the medical services to the remote areas remotely.

The remainder of the paper is organized as follow: Section 2 presents the related work, Section 3 illustrates the system overview, Section 4 describes the system implementation and in Section 5 presents the conclusions and future work.

Table 1. Main common functions pregnant apps

\begin{tabular}{|c|l|}
\hline$\#$ & \multicolumn{1}{|c|}{ Functions } \\
\hline 1 & Expected day delivery \\
\hline 2 & Kick Counter \\
\hline 3 & Personal Diary \\
\hline 4 & Pregnancy Exercise \\
\hline 5 & Weight Tracker \\
\hline 6 & Contraction Timer \\
\hline 7 & Pregnancy Nutrition \\
\hline 8 & Pregnancy Vitamins and Medications \\
\hline 9 & Pregnancy Forum \\
\hline 10 & Daily and weekly information, image and videos \\
\hline 11 & Pregnancy Appointments \\
\hline 12 & Mood Tracker \\
\hline 13 & Countdown \\
\hline 14 & Scientific Articles \\
\hline 15 & BABYBUMP SHOP \\
\hline 16 & Hospital bag checklist \\
\hline 17 & Information about your baby after delivery \\
\hline 18 & Pregnancy Vitamins and Medications \\
\hline
\end{tabular}




\section{System Overview}

The pregnancy application, showing in this paper, is a remote monitoring system designed to encourage pregnant women in Saudi Arabia to increase their physical activities, pregnancy awareness, exchange their experiences with each other's, and communicate with their doctors effectively. Additionally, it empowers doctors to deliver information to their pregnant using modern technology. The architecture of the system is developed to facilitate the main features that eliminate challenges associated with pregnancy worldwide and specifically in Saudi Arabia. These include poor pregnancy awareness, lack of motivation for physical activity and inability to count baby kicks. Furthermore, it also is designed to provide various features that create an attractive environment for pregnant women and cover the lack of Obstetrics and Gynecology Clinic specially in remote areas of Saudi. The architecture for the developed system is show in figure 1 .

\section{Pregnancy awareness / remote monitoring provider component}

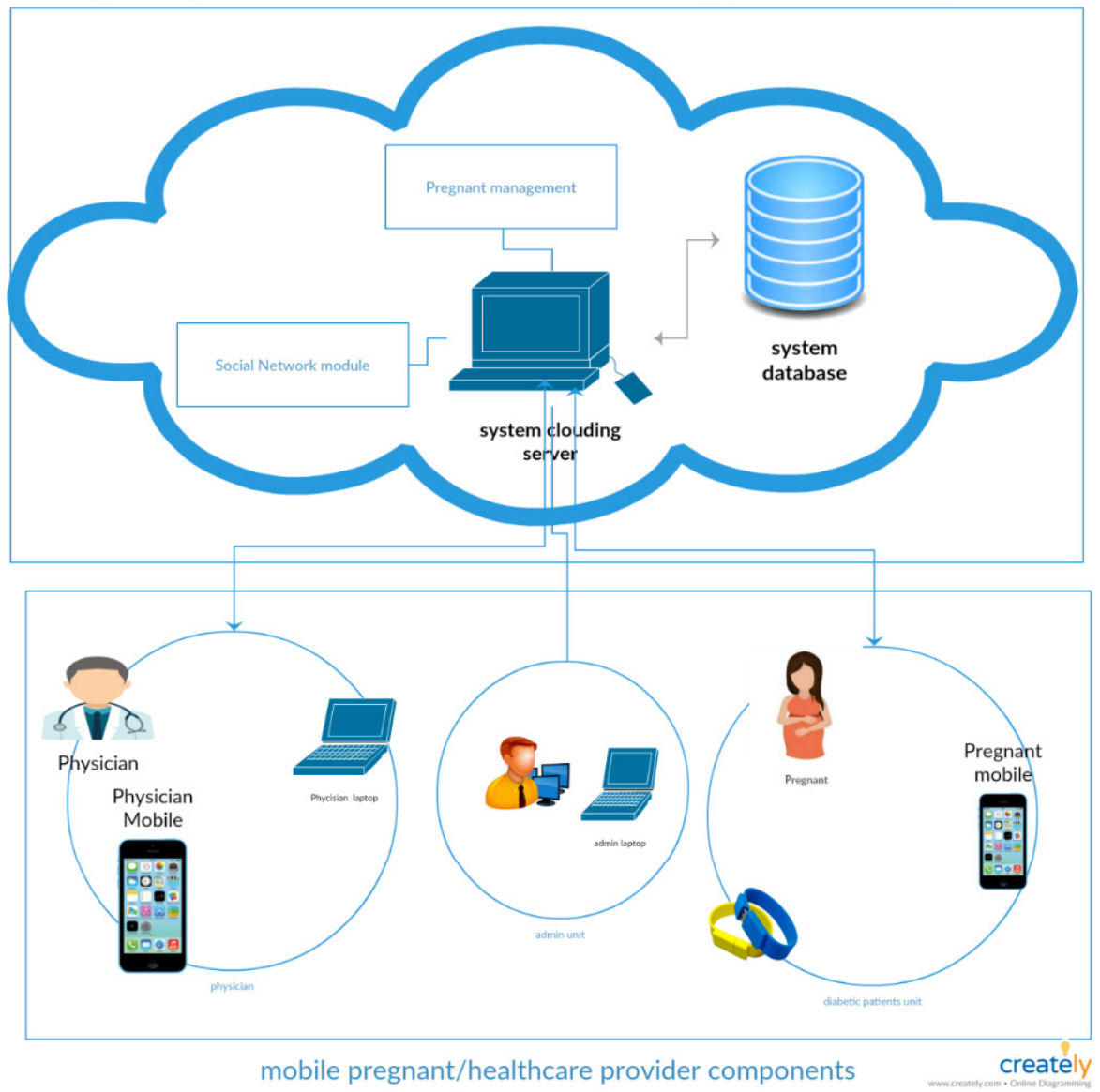

Fig. 1. System Architecture 
Therefore, the system architecture is divided into two main components:

Mobile pregnant/healthcare provider components: This station includes the mobile end of the system. It is implemented using smart phone platform and use of the Internet of Things (IoT) technology. This component covers most of the interaction units of the system. It also comprises of three units:

HealthCare provider unit. This unit consists of a friendly web interface and mobile application version that helps nurses or clinicians involved in the diagnosis to create awareness and conduct follow-ups of the pregnant. It is a private social network for pregnancy community, which enables the physician to communicate with pregnant women remotely. It also allows the pregnant mothers to interact with each other too. The physicians can use the system to educate the pregnant women using this modern technology. Physicians can post messages, upload images, videos and communicate with their pregnant women privately using the private SMS message tool. To save people's lives and guarantee tope quality of written posts and uploaded media, the physician is allowed to delete incorrect pregnancy posts, image and video.

It also furnishes the physician with full details about the pregnant woman's profile including physical activity using wrist bond and the frequency of baby kicks using the belt fitted with body touch sensor.

Pregnancy unit. This unit typically includes:

5. A smart mobile phone

6. Wrist bond

7. Kick counter belt sensors

In this unit, the pregnant women can participate in social networking community, read and share information, as well as develop experiences about the pregnancy lifestyle as provided by the doctors/nurses and other pregnant in the community. The pregnant mothers are encouraged to read the content and post comments and reviews related to the doctors' posts.

The pregnant women interact or use the system via a friendly web interface and mobile application that includes a social networking platform and data management system, which presents pregnant' personal medical profile. Moreover, the pregnant women will have access to all functionalities including following others. For a private issue, the pregnant mothers can communicate with each other and with their doctors through the private SMS tool available in the system.

In addition, the wrist bond will record the number of steps that the pregnant lady walked during the day while the kick belt sensor will record the number of baby kicks. The information will be recorded and stored in the database through WIFI. It will be available for the clinicians through the cloud technology.

The admin components: Admin components refer to the technical support. The admin is accountable for the system's repair and maintenance. The technical personnel monitor the technical parts of the system and is also responsible for maintaining the backup of the data collected via the system. The admin has full access to the users profile and, hence, can delete user, edit users profile and validate physician accounts.

Management provider component: This component represents the back-end operations, which mainly involves the process of collecting information or data and 
storing it in the database. The data is, subsequently, used by s other modules such as the awareness module, management module, the front-end patient, as well as the clinician mobile units. This component includes Database module, which stores information related to data related to individual pregnant mothers and records related to the laboratory examination results. It may also store specialist nurses/doctor comments on the pregnancy measurements standard. This component includes two modules namely:

Social Network module: The social network module is an important functionality of the system. It is designed to empower the pregnant women to develop increased awareness about pregnancy. The idea is to enhance their knowledge through social networking. In this module there is a private social network for pregnant community and their doctors. The medical staff will play the main role in this module. They will prepare a number of posts, images and videos of information regarding pregnancy. In addition, they will also answer pregnancy-related questions as well as any queries related to diverse diseases. All features delivered by this module are described in the users' units above.

Management module: These functions of this module include:

a) Walking counter: It is important to clarify what we mean by physical activity. PA refers to the voluntary body movements that increase energy consumption, like leisure time and occupational activity, during pregnancy (4). In this unit, pregnant women will be motivated to reach a weekly target of steps. The number of steps will be calculated through our developed wrist bond. Wrist bond is easier than using the accelerometer in the mobile device because it can be placed on the wrist of the pregnant woman all the time. Additionally, wrist bond sends the recorded data to the cloud technology via a wireless connection. Moreover, it is less expensive because it costs 20 USD unlike the expensive Apple watch.

b) Expected Delivery Date. Both the physicians and pregnant women should have knowledge about the expected delivery time. Hence, the system will calculate the expected delivery day based on available data in the system. The system will reflect these details in the pregnant woman's profile.

c) Kick counter: In the third trimester, doctors normally enquire about the movement of the fetus in terms of frequency and duration to determine the health of the fetus. Kick counting is an easy way to monitor and record the movement of the unborn baby. A belt fitted with a sensor, which can record baby kick count is under development using Flex sensor technology, figure 2. We aimed to record the kick count and transfer it to the cloud server using WIFI technology. When complete, the system will alert the doctor and the pregnant mothers to take action in the absence of fetal movement. The physician will set up the time period of monitoring the baby movement using the system.

d) Mood Tracker: Every pregnant woman will receive a message every day and will be expected to make a response regarding her mood by selecting a number between 1 and 10. 1 represents high (positive) mood while 10 represents low (negative) mood. The daily response will be recorded in her profile using date and time. 
e) Pregnancy Vitamins and Medications: As obviously known, these details are essential for supporting the decision-making operations of the physicians. Therefore the pregnant women must record their vitamins and medication. The system will allow pregnant women to record their vitamins and medication, which doctor can access via their profiles.

f) Weight tracker: The weight of the pregnant women will be recorded weekly and saved in her profile.

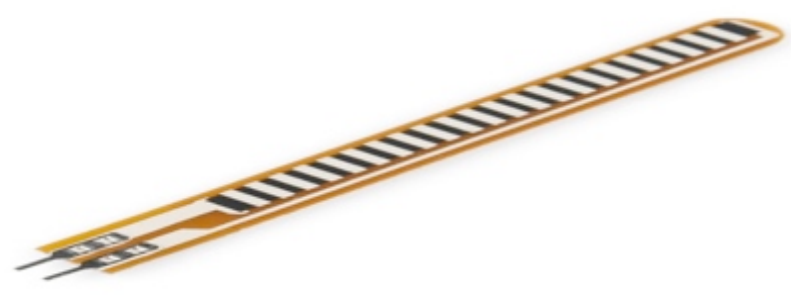

Fig. 2. Flex Sensor

\section{System Implementation and discussion}

A prototype system was developed at the faculty of computing and information technology at the University of Tabuk, Tabuk, Saudi Arabia using tool such as PHP and MySQL for the web application, Java for android and SWIFT for the IOS, Arduino Mega, 3D Printer, Flex Sensor, and Spark Fun Triple Axis Accelerometer Breakout - ADXL335.

Researchers tested the system and confirmed that the system is capable of carrying out all the functional requirements. They also demonstrated that the system is capable of transmitting medical data from a remote pregnant woman to the host server. The system allows the pregnant mothers and physicians to share information remotely. The mobile application home screen is shown in figure 3 .

As presented in figure 4, the system mobile application allows the pregnant women and the specialist nurse/doctors to share their profiles. Furthermore, the application allows the pregnant women and the clinicians to communicate with each other privately via the free application's SMS tool.

Figure 5 shows the user control panel. The specialist nurse/doctor will add videos, album of pictures and access the patient's profile remotely using the mobile application. 
Paper-A smart mobile pregnancy management and awareness system for Saudi Arabia

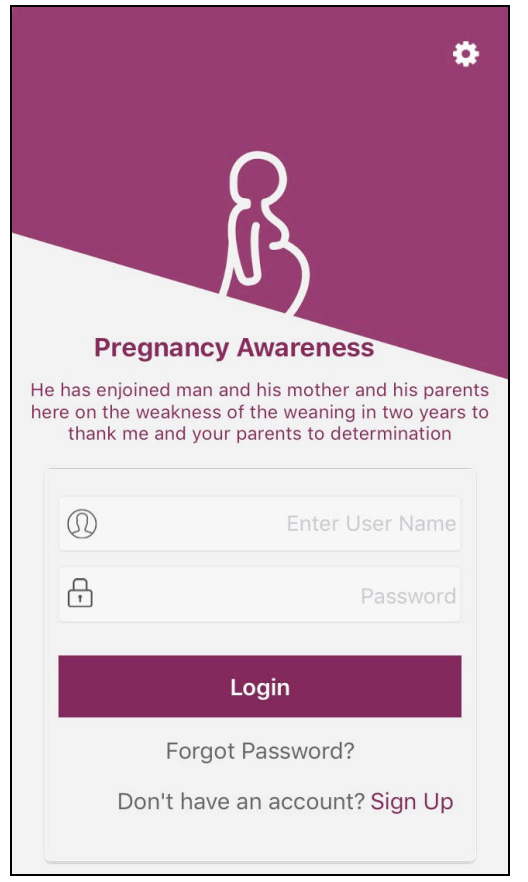

Fig. 3. Snap screen of the system mobile application

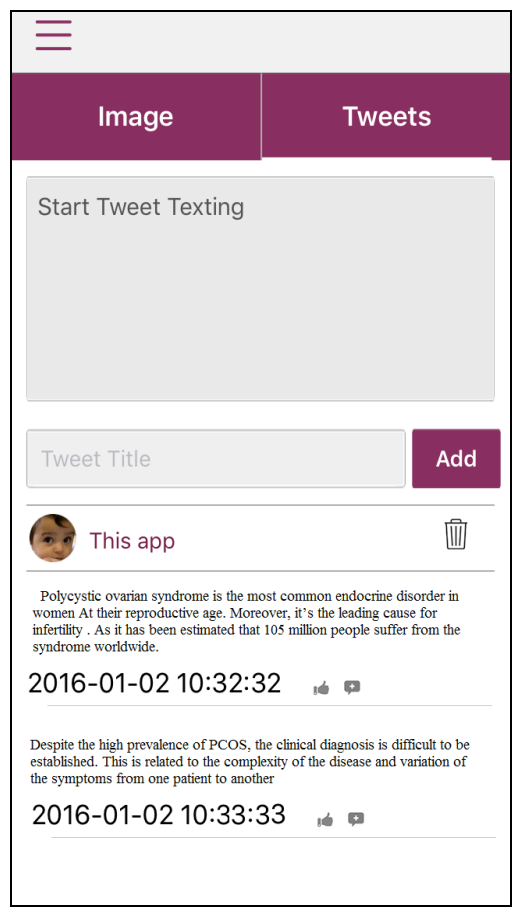

Fig. 4. Snap screen of the application Doctors' posts

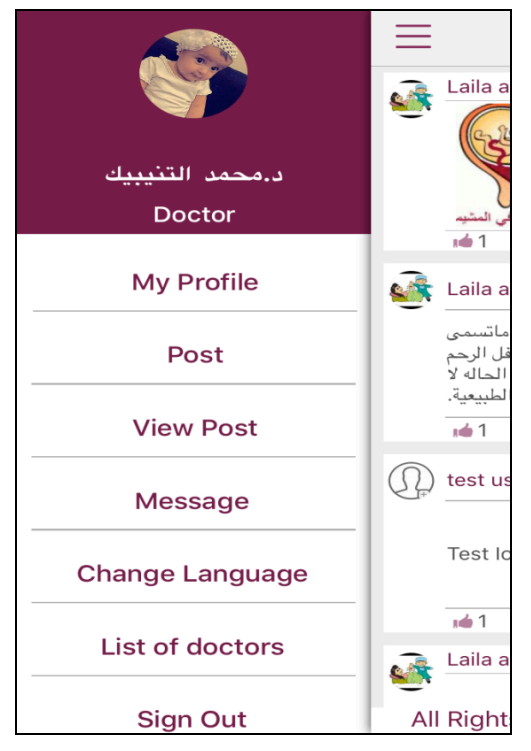

Fig. 5. The user control panel 
Moreover, the wrist bond is built using the SparkFun Triple Axis Accelerometer Breakout - ADXL335, figure 6. The ADXL335 is a triple axis MEMS accelerometer with new features including (1) extremely low noise and (2) power consumption of only $320 \mathrm{uA}$. Moreover, it has a full sensing range of $+/-3 \mathrm{~g}$. It is programmed using Arduino and $\mathrm{C}++$. It comes in various colors. It sends data to the cloud server remotely.

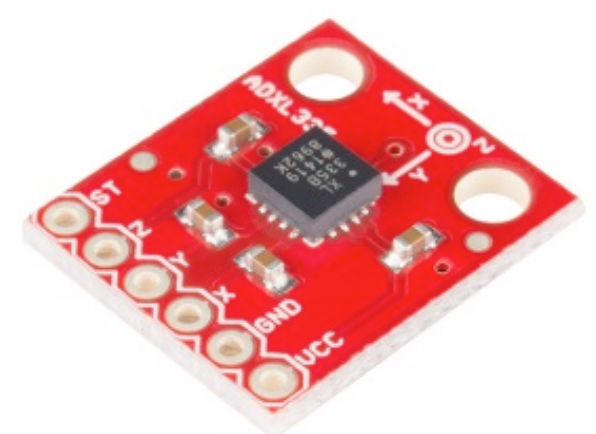

Fig. 6. SparkFun Triple Axis Accelerometer Breakout

The researchers tried to develop an innovative mobile pregnancy awareness and monitoring system with all features based on the literature of related work, previous studies, and existing pregnancy applications in iTunes and Play Store. The objective was to provide cost-effective technology.

In the reviewed applications and studies, the mobile embedded accelerometer requires the pregnant to carry the mobile with her at all times. The technique was effective because it reached the predetermined target. However, there are several limitations that diminish its effectiveness. In particular, these include mobile battery consumption and the need to carry the mobile while doing the exercise. As a result, the researchers feels that using smart watch or wrist bond could be easier, effective, and flexible. Study (8) used the FitBit to measure the physical activity using steps counting. The technique involved counting the number of steps. It was effective due to the ease of carrying the mobile devises to any place at all times. However, the strategy is not cost-effective given that the cost of the used FitB is around 1400 Saudi Riyal while the cost of a smart watch like Apple watch is roughly 2400 Saudi Riyal. Hence, the researcher opted to develop a new wrist bond that will cost utmost 50 Saudi riyal. Such a device would be cheaper for the Saudi people. Moreover, in the same study (8) they used chargeable SMS messages, so the researcher developed free- SMS messages based on push notifications.

The population of Saudi nationals using social networking sites, such as Twitter and Facebook, is high. For instance, the populace of Saudi citizens using twitter in 2016 exceeded the 5 million mark by far (14). As twitter users from Saudi Arabia, we have noticed that a significant number of tweets sent from the Saudi and Arabic world are tweeting about health and medicine. Majority of those accounts are not authenticated by Twitter and, hence, it is difficult to validate the content. It is shocking an 
unethical to note that most of these tweets contain unhealthy information, which might put people lives in danger. It is difficult to close the accounts. Thus, we can only block and report them to Twitter, Inc. for closure. Unfortunately, such a process takes a long time.

Therefore, the researchers can develop a social network similar to Twitter to guarantee validity of every content posted in the platform. The persons masquerading as doctors ought to be authenticated by the system administrator. Doctors should not have the liberty or power to tweet until they gain authentication by the system admin. Authentication should confirm the identity and qualification of the platform users. Moreover, the network administrator should have the power and privilege to remove any incorrect content related to pregnancy.

The main feature of the new system is that all features that are distributed between the reviewed applications are under one umbrella. The baby count belt is under development. We initially get good result and the development is ongoing.

\section{Conclusion and Future Work}

The smart mobile pregnancy system is designed to promote pregnancy awareness and remote monitoring. It is based on the state-of-the-art technological infrastructures. As such, it is presented to achieve intensive monitoring at home, optimized treatment, as well as a normal lifestyle for the pregnant mothers in Saudi Arabia without restricting their daily lives. Moreover, the system is designed to deliver relevant information regarding pregnancy to help the women avoid unnecessary diseases.

Future research should aim to evaluate the system by running it in the KSA. The implementation of the system should be following the regulation of national ethical approval at the University of Tabuk, Saudi Arabia. We project that the trial will last 9 months. Therefore, this long-term study will evaluate the effectiveness of using the Internet of Things (IoT) to improve pregnancy management and awareness.

Upon completion of this long-term study, it is important to conduct thorough testing. The questionnaire can help the researchers to study the system's usability solely based on the views of the pregnant women and clinicians. To evaluate the impact of the system on pregnancy awareness and management, the questionnaire will be distributed to both pregnant mothers and clinicians before and after the trial. The researchers will conduct extensive comparisons between the two results. Meanwhile, the pregnancy knowledge test will be conducted using a standardized questionnaire before and after the study. When the trial is complete, the results will be studied and analysed using statistical software such as SPSS.

\section{References}

[1] The World Factbook - Central Intelligence Agency [Internet]. [cited 2018 May 18]. Available from: https://www.cia.gov/library/publications/the-world-factbook/fields/ 2054.html 
[2] Huberty J, Rowedder L, Hekler E, Adams M, Hanigan E, McClain D, et al. Development and design of an intervention to improve physical activity in pregnant women using Text4baby. Transl Behav Med. 2016 Jun;6(2):285-94. https://doi.org/10.1007/s13142015-0339-7

[3] Doran F, Davis K. Factors that influence physical activity for pregnant and postpartum women and implications for primary care. Aust J Prim Health. 2011;17(1):79-85. https://doi.org/10.1071/PY10036

[4] Leite CF, do Nascimento SL, Helmo FR, Dos Reis Monteiro MLG, Dos Reis MA, Corrêa RRM. An overview of maternal and fetal short and long-term impact of physical activity during pregnancy. Arch Gynecol Obstet. 2017 Feb;295(2):273-83. https://doi.org/10.1007/ s00404-016-4204-9

[5] Melzer K, Schutz Y, Soehnchen N, Othenin-Girard V, Martinez de Tejada B, Irion O, et al. Effects of recommended levels of physical activity on pregnancy outcomes. Am J Obstet Gynecol. 2010 Mar;202(3):266.e1-6. https://doi.org/10.1016/j.ajog.2009.10.876

[6] May LE, Glaros A, Yeh H-W, Clapp JF, Gustafson KM. Aerobic exercise during pregnancy influences fetal cardiac autonomic control of heart rate and heart rate variability. Early Hum Dev. 2010 Apr;86(4):213-7. https://doi.org/10.1016/j.earlhumdev.2010.03.002

[7] Evenson KR, Savitz DA, Huston SL. Leisure-time physical activity among pregnant women in the US. Paediatr Perinat Epidemiol. 2004 Nov;18(6):400-7. https://doi.org/10.1111/ j.1365-3016.2004.00595.x

[8] Huberty JL, Buman MP, Leiferman JA, Bushar J, Hekler EB, Adams MA. Dose and timing of text messages for increasing physical activity among pregnant women: a randomized controlled trial. Transl Behav Med. 2017 Jun;7(2):212-23. https://doi.org/10.1007/ s13142-016-0445-1

[9] Istepanian R, Laxminarayan S, Pattichis CS, editors. M-Health: Emerging Mobile Health Systems [Internet]. Springer US; 2006 [cited 2018 May 18]. (Topics in Biomedical Engineering. International Book Series). Available from: http://www.springer.com/gp/book/ $9780387265582 \mathrm{https}: / /$ doi.org/10.1007/b137697

[10] Donner J, Mechael P, editors. mHealth in Practice: Mobile technology for health promotion in the developing world. 1 edition. London: Bloomsbury Academic; 2013. 208 p.

[11] Olla P. Mobile Health Solutions for Biomedical Applications. 1 edition. Tan J, editor. Hershey Pa.: Medical Information Science Reference; 2009. 366 p. https://doi.org/10.4018/978-1-60566-332-6

[12] Alotaibi MM, Istepanian R, Philip N. A mobile diabetes management and educational system for type-2 diabetics in Saudi Arabia (SAED). mHealth. 2016;2:33. https://doi.org/10.21037/mhealth.2016.08.01

[13] Choi J, Lee JH, Vittinghoff E, Fukuoka Y. mHealth Physical Activity Intervention: A Randomized Pilot Study in Physically Inactive Pregnant Women. Matern Child Health J. 2016 May;20(5):1091-101. https://doi.org/10.1007/s10995-015-1895-7

[14] Saudi Arabia: number of Twitter users 2016 | Statistic [Internet]. Statista. [cited 2018 May 18]. Available from: https://www.statista.com/statistics/558404/number-of-twitter-usersin-saudi-arabia/

\section{$7 \quad$ Acknowledgements}

Authors gratefully acknowledge the support and assistance of the Club of Robotics, Faculty of Computing and information Technology, University of Tabuk, Saudi Arabia. 


\section{Authors}

Mohammed Alotaibi is an assistant professor of Bio-Informatics at Faculty of Computers and Information Technology, University of Tabuk, Tabuk, Saudi Arabia. $\mathrm{He}$ is also a member of Women's health Informatics research group.

Maram Albalawi is a lecturer in Software Engineering at Faculty of Computers and Information Technology, University of Tabuk, Tabuk, Saudi Arabia. She is also a member of Women's health Informatics research group.

Layan Alwakeel is a lecturer in Software Engineering at Faculty of Computers and Information Technology, University of Tabuk, Tabuk, Saudi Arabia. She is also a member of Women's health Informatics research group.

Article submitted 09 June 2018. Final acceptance 16 july 2018. Final version published as published by the authors. 